\title{
Lagrangian Micromixing Models for Concentration Fluctuations: An Overview
}

\author{
${ }^{1}$ Andrea Amicarelli, ${ }^{2}$ Giovanni Leuzzi and ${ }^{2}$ Paolo Monti \\ ${ }^{1}$ Department of Environment and Sustainable Development (ASV), Via Rubattino, 54, 20134 Milan, Italy \\ ${ }^{2}$ DICEA, Universita di Roma "La Sapienza”, Via Eudossiana 18, 00184 Roma, Italy
}

Received 2012-06-20, Revised 2012-07-26; Accepted 2012-12-20

\begin{abstract}
This study presents a brief overview of the main features, theoretical formulations and validation tests of some Lagrangian micromixing models, currently used for estimations of the ensemble mean and the turbulent fluctuations of concentration. Their application fields regards several pollutant dispersion phenomena such as: accidents (power or production plants, terroristic attacks, hydrocarbons storage and transport, extraordinary emissions), odours (power plants and energy production from waste resourcescompost, dumps, incinerators, biogas storage and smokes-, high enthalpy geothermic plants-sulfide hydrogen-, animal farms), micro-scale dispersion from continuous or spot emissions (traffic pollutants, power and production plants, dispersion in aquatic environments...), industrial processes (combustion, pollutant treatment,...), strong non-linear relationship between concentration and damage (inflammable substances, explosions,..), reactions depending on instantaneous concentrations.
\end{abstract}

Keywords: Micro-Scale Dispersion, Odours, Accidents, Pollutant Dispersion, Micromixing, Macromixing, Concentration Fluctuations, Lagrangian Micromixing Models

\section{INTRODUCTION}

Turbulent fluctuations of concentration play a key role in several dispersion phenomena, involving water or atmospheric pollutants, mainly where the pollutant fly time $\left(\mathrm{t}_{\mathrm{f}}\right)$ is smaller than the integral Lagrangian time scale (micro-scale dispersion). In particular, concentration fluctuations are relevant when modelling the transformation processes of several reactions, as these normally depend on the instantaneous concentrations rather than their mean values. Under these conditions, the correlation of the concentrations of reactive pollutants may be not negligible (second order kinetics reactions). Furthermore, concentration fluctuations are of particular interest when damage is strongly non-linear with respect to concentration, especially if the influence of its peaks or minima is crucial.

In this context the mean concentration, defined in terms of Reynolds' average concentration (commonly referred to as "concentration"), is generally not adequate to represent the time and spatial evolutions of the instantaneous concentration field. Numerical modelling should then try to reproduce concentration fluctuations in terms of probability density function (pdf) of concentration ( $\mathrm{f}_{\mathrm{C}}$ or concentration pdf), or at least its first statistical moments.

Several dispersion phenomena would then need to be studied in terms of concentration fluctuations:

- Accidents: Pollutant dispersion in atmosphere or water bodies related to emissions from power or production plants, terroristic attacks, hydrocarbons storage and transport, inflammable substances, explosions

- Odours: Ollutants emitted from power plants and waste-to-energy processes (composting, dumps, incinerators, biogas storage and smokes), highenthalpy geothermal plants (hydrogen sulfide) or animal farms

Corresponding Author: Andrea Amicarelli, Department of Environment and Sustainable Development (ASV), Via Rubattino, 54, 20134 Milan, Italy 
- Micro-scale: Micro-scale dispersion from continuous or spot emissions (from energy or production power plants vehicular traffic...)

- Combustion processes: Combustion processes motivated the first development of micromixing schemes

Several numerical schemes have been developed in order to evaluate concentration fluctuations, based on Direct Numerical Simulations (DNS), Large Eddy Simulations (LES), pdf models, Reynolds' Average Navier-Stokes models (RANS) and "fixed pdf" models (Amicarelli, 2009). In this context Lagrangian micromixing models seem to represent one of the most efficient approaches in terms of both accuracy and computational costs.

Lagrangian micromixing models evaluate the mean and the higher moments of concentration, coupling a macromixing scheme and a micromixing scheme. The first evaluates the trajectories of fictitious fluid particles, representing the turbulent transporting flow, the latter simulates the molecular diffusion processes and the pollutant transformations. The resulting governing equations, someway constrained by the balance equation of the mean concentration, represent a Lagrangian stochastic system.

Several parameters quantify the role of the concentration fluctuations and refer to $\mathrm{f}_{\mathrm{C}}$ : Mean $(\overline{\mathrm{C}})$, standard deviation $\left(\sigma_{\mathrm{C}}\right)$, intensity of fluctuations $\left(\mathrm{i}_{\mathrm{C}}=\overline{\mathrm{C}} / \sigma_{\mathrm{C}}\right)$, skewness $\left(\mathrm{S}_{\mathrm{C}}\right)$, kurtosis $\left(\mathrm{K}_{\mathrm{C}}\right)$, the 99th percentile of $\mathrm{f}_{\mathrm{C}}$ or peak concentration $\left(\mathrm{C}_{99}\right)$ and the intermittency factor $(\chi)$, the latter defined as the probability of positive concentrations.

Several experimental studies, some of which reported in Table 1, underlines the role of concentration fluctuations at the micro-scale. This study continuously refers to the minor publication (Amicarelli, 2009). After a brief introduction (sec 1), sec 2 shows the main characteristics of the numerical formulations of these models, while sec 3 presents an overview of some reference Lagrangian micromixing studies, describing their numerical settings and results. Second 4 finally reports the general conclusions of the study. In the following, over-bars denote Reynolds' averages and primes turbulent fluctuations, according to the Reynolds' decomposition.

\section{NUMERICAL FORMULATIONS}

Lagrangian micromixing models couple a macromixing scheme, which estimates the particle trajectories, with a micromixing scheme, which takes into account molecular diffusion processes and pollutant transformations.

A generic and simple algorithm for Lagrangian micromixing models could be synthesized as follows. Particles are released all over the numerical domain, both at the source locations (non-null concentration) and elsewhere (null concentration or other initial values). At each time step two numerical and sequential phases can be identified: the macromixing and the micromixing ones. During the first phase, particles move according to the macromixing scheme and the mean concentrations are computed. During the latter one, particle concentrations evolve according to the micromixing scheme. Further, the particles leaving the domain are replaced by new particles generated at the inflow sections, in order to continuously model the whole turbulent flow. We notice that the particles should represent the motion of the whole fluid mass inside the domain, for several realization of the same experiment. Under the hypothesis of Reynolds' decomposition in fact, Lagrangian micromixing models simultaneously reproduce a great number of realizations of the turbulent flow. The number of particles should be then enough large in order to guarantee that in each cell of the underlying grid all the computed concentration statistics are enough robust. This underlying or background grid is not a computational mesh used to solve the governing equations. It represents in fact a domain partition, which just defines the cells where to count the statistics of the particle concentrations.

The above generic algorithm is normally modified, to minimize the computational costs, according to the particular phenomenon to be simulated, as described.

Some general considerations about macromixing and micromixing schemes are hereafter discussed.

In the case of high Reynolds numbers (turbulent regimes), molecular diffusion does not affect concentration mean (Pope, 1998). This involves that we can neglect molecular diffusion when writing the Reynolds' average of the balance equation of $\mathrm{C}$ Equation 1:

$$
\frac{\overline{\mathrm{dC}}}{\mathrm{dt}}=0
$$

We are then allowed to use conservative particles (with constant concentration along the trajectory) to represent passive pollutants, as they satisfy (1), when averaging according to Reynolds Equation 2:

$$
\frac{\mathrm{dC}}{\mathrm{dt}}=0 \Rightarrow \quad \frac{\overline{\mathrm{dC}}}{\mathrm{dt}}=0
$$


Table 1. Underlines the role of concentration fluctuations at the micro-scale

\begin{tabular}{lllcc}
\hline Year & Authors & Boundary Layer (BL) type & $\mathrm{i}_{\mathrm{C}}$ & $\mathrm{C}_{99} / \overline{\mathrm{C}}$ \\
\hline 1967 & Csanady (1967) & Report of experimental studies of other works & $/$ & $2-700$ \\
1982 & Fackrell and Robins (1982) & Wind tunnel Neutral BL (NBL) & $0-2.5$ \\
1986 & Mah and Warhaft (1986) & Grid turbulence, wind tunnel & $0-1$ & $/$ \\
1994 & Yee et al. (1994) & Stable BL & $0.8-13$ & $3-670$ \\
& & wind tunnel NBL with building & $1-47$ & $2-96$ \\
1995 & Mylne et al. (1995) & NBL & $1-9$ & $/$ \\
1997 & Chatwin and Robinson (1997) & wind tunnel, buildings, passive and heavy gases & $0-4$ & $/$ \\
1997 & Chatwin et al. (1997) & Report of results from other works & $3-6$ & $/$ \\
1999 & Pavageau and Schatzmann (1999) & wind tunnel, buildings & $0.2-3$ & $2-22$ \\
2004 & Yee and Biltoft (2004) & MUST experiment, NBL and SBL, with buildings & $0.2-5$ & $14-25$ \\
2007 & Bezpalcova (2007) & MUST wind tunnel experiment, NBL, buildings & $0-18$ & $/$ \\
2011 & Klein et al. (2011) & Joint Urban 2003 project (urban BL) - full scale & $0-4$ & $/$ \\
2011 & Klein and Young (2011) & Joint Urban 2003 project (urban BL) - wind tunnel & $0-5$ & $/$ \\
\hline
\end{tabular}

Analogously, the mean concentration conditioned to the Lagrangian velocity vector $\langle\mathrm{C} \mid \underline{\mathrm{U}}\rangle$ is independent of molecular diffusion (in particular (3) implies (2) Equation 3:

$$
\frac{\mathrm{d}\langle\mathrm{C} \mid \underline{\mathrm{U}}\rangle}{\mathrm{dt}}=0
$$

Macromixing schemes can then model mean concentrations of passive pollutants thanks to the property (2), provided that their trajectories are correctly reproduced, as described. The resulting particle concentrations however are not representative of instantaneous concentrations, which are affected by molecular diffusion processes.

Macromixing schemes in fact would provide highly overestimated concentration fluctuations. Further even the mean concentration of reactive pollutants cannot be properly modelled, if the transformations depend on concentration fluctuations (as for second order kinetics reactions) and not on the concentration means.

Lagrangian micromixing models than need to couple a micromixing scheme to a macromixing scheme, in order to represent molecular diffusion phenomena and pollutant transformations.

Pope (1998) lists the main properties a micromixing model should fill. It has:

- To reduce concentration fluctuations, as they would result from a macromixing model

- Not to influence the mean, the conditional mean and the turbulent flux of concentration for passive pollutants

- To provide a Gaussian $\mathrm{f}_{\mathrm{C}}$ if the concentration field is homogeneous

- To provide concentration values in a limited range (not negative, nor higher than $\mathrm{C}$ at source position)
The last two properties can appear in contrast, but $\mathrm{f}_{\mathrm{C}}$ can be eventually cut at the origin and at the maximum value of $\mathrm{C}$.

A main reference for concentration fluctuations is the balance equation of the concentration variance in turbulent regimes ( $\underline{\underline{u}}_{\underline{i}}$ is the component of the Eulerian velocity with respect to the $\mathrm{x}_{\mathrm{i}}$-axis, $\mathrm{D}_{\mathrm{M}}$ the molecular diffusion, $\mathrm{T}$ the reactive term of the balance equation of C; (Csanady, 1967) Equation 4:

$$
\begin{aligned}
& \frac{\partial \sigma_{\mathrm{C}}^{2}}{\partial \mathrm{t}}+\overline{\mathrm{u}_{\mathrm{i}} \frac{\partial \sigma_{\mathrm{C}}^{2}}{\partial \mathrm{x}_{\mathrm{i}}}}+\overline{\mathrm{u}_{\mathrm{i}}{ }^{\prime} \frac{\partial\left(\mathrm{C}^{\prime}\right)^{2}}{\partial \mathrm{x}_{\mathrm{i}}}}=-2 \overline{\mathrm{u}_{\mathrm{i}} \mathrm{C}^{\prime}} \frac{\partial \overline{\mathrm{C}}}{\partial \mathrm{x}_{\mathrm{i}}} \\
& -2 \mathrm{D}_{\mathrm{M}} \overline{\left(\frac{\partial \mathrm{C}^{\prime}}{\partial \mathrm{x}_{\mathrm{i}}}\right)^{2}}+2 \overline{\mathrm{C}^{\prime} \mathrm{T}^{\prime}}
\end{aligned}
$$

A micromixing scheme only influences the last two terms of (4), the dissipation rate of the concentration variance $\left(\varepsilon_{\mathrm{C}}\right)$ and the reactive term $\left(\overline{\mathrm{R}_{2}}\right)$, respectively. We note that $\varepsilon_{\mathrm{C}}$ depends on the Eulerian derivatives of concentration fluctuations, which needs adequate formulations in Lagrangian modelling.

According to the balance equations for the mean concentration and the concentration fluctuation Equation 5:

$\frac{\overline{\mathrm{dC}}}{\mathrm{dt}}=\mathrm{D}_{\mathrm{M}} \frac{\partial^{2} \overline{\mathrm{C}}}{\partial \mathrm{x}_{\mathrm{i}}^{2}}+\overline{\mathrm{T}}, \frac{\mathrm{dC^{ \prime }}}{\mathrm{dt}}=\mathrm{D}_{\mathrm{M}} \frac{\partial^{2} \mathrm{C}^{\prime}}{\partial \mathrm{x}_{\mathrm{i}}^{2}}+\mathrm{T}^{\prime}$

From these relations and (Amicarelli, 2009) follows Equation 6:

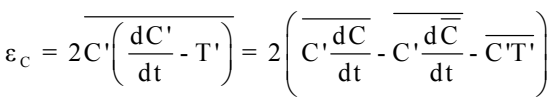

$$
\begin{aligned}
& =2\left(\overline{C^{\prime}} \frac{\mathrm{dC}}{\mathrm{dt}}-\overline{\mathrm{C}^{\prime} \mathrm{T}^{\prime}}\right)
\end{aligned}
$$


If the reactive term is negligible the micromixing scheme should correctly model the term Equation 7:

$$
\varepsilon_{\mathrm{C}}=2 \overline{\mathrm{C}^{\prime}} \overline{\mathrm{dC}}
$$

On the other hand, when present, the reactive terms can be expressed as functions of instantaneous concentrations (as will be highlighted).

\subsection{The Macromixing Schemes}

The most rigorous macromixing scheme, used to estimate particle trajectories, was developed by Thomson (1987). Lagrangian velocities $\underline{\mathrm{U}}=(\mathrm{U}, \mathrm{V}, \mathrm{W})$ are updated every time step (whose duration is dt) Equation 8:

$$
\begin{aligned}
& \underline{\mathrm{U}}\left(\mathrm{t}+\mathrm{dt} ; \underline{\mathrm{x}}_{0}, \mathrm{t}_{0}\right)=\underline{\mathrm{U}}\left(\mathrm{t} ; \underline{\mathrm{x}_{0}}, \mathrm{t}_{0}\right)+\underline{\mathrm{dU}}\left(\underline{\mathrm{t} ;} \underline{\mathrm{x}}_{0}, \mathrm{t}_{0}\right), \\
& \underline{\mathrm{U}}\left(\underline{\mathrm{x}_{0}}, \mathrm{t}_{0}\right)=\underline{\mathrm{U}_{0}}
\end{aligned}
$$

The subscript "0" denotes the initial conditions. Integrating (8) in time we can derive particle positions $\underline{\mathrm{X}}$ $=(\mathrm{X}, \mathrm{Y}, \mathrm{Z})$ Equation 9:

$$
\begin{aligned}
& \underline{\mathrm{X}}\left(\mathrm{t}+\mathrm{dt} ; \underline{\mathrm{x}_{0}}, \mathrm{t}_{0}\right)=\underline{\mathrm{X}}\left(\mathrm{t} ; \underline{\mathrm{x}_{0}}, \mathrm{t}_{0}\right)+\underline{\mathrm{U}}\left(\mathrm{t} ; \underline{\mathrm{x}_{0}}, \mathrm{t}_{0}\right) \mathrm{dt}, \\
& \underline{\mathrm{X}}\left(\underline{\mathrm{x}_{0}}, \mathrm{t}_{0}\right)=\underline{X_{0}}
\end{aligned}
$$

We even need to define the integral Lagrangian time $\operatorname{scale}\left(\mathrm{T}_{\mathrm{L}, \mathrm{ij}}\right)$, which represent the integral of the autocorrelation matrix of the Lagrangian velocities $\left(\sigma_{\mathrm{u}, \mathrm{i}}\right.$ are the standard deviations of velocity) Equation 10:

$$
\mathrm{T}_{\mathrm{L}, \mathrm{j}} \equiv \int_{\mathrm{t}_{0}}^{\infty} \frac{\overline{\mathrm{U}_{\mathrm{i}}\left(\mathrm{t} ; \underline{\mathrm{x}}_{0}, \mathrm{t}_{0}\right) \mathrm{U}_{\mathrm{j}}\left(\mathrm{t}_{0} ; \underline{\mathrm{x}}_{0}, \mathrm{t}_{0}\right)}}{\sigma_{\mathrm{u}, \mathrm{i}}(\underline{\mathrm{X}}(\mathrm{t}), \mathrm{t}) \sigma_{\mathrm{u}, \mathrm{j}}\left(\underline{\mathrm{x}_{0}}, \mathrm{t}_{0}\right)} \mathrm{dt}
$$

Because the lack of data regarding the off-diagonal terms, this parameter $T_{\mathrm{L}, \mathrm{ij}}$ is sometimes used as a vector and commonly as a scalar $\left(\mathrm{T}_{\mathrm{L}}\right)$. The particle velocity at time $t$ is correlated with the velocity values of the same particles in the immediately previous period, up to a certain time, approximately equal to $\left(\mathrm{t}-\mathrm{T}_{\mathrm{L}}\right)$. Fixed a generic point in space the norm of $\underline{T}_{L}$ represents the time scale of the largest turbulent vortices passing for the cited point.

Thomson (1987) then uses the relation which links $\underline{T}_{L}$ with the rate of dissipation of the turbulent kinetic energy $\varepsilon$ and the standard deviations of the velocity components Equation 11:

$\mathrm{T}_{\mathrm{L}_{\mathrm{i}}}=\frac{2 \sigma_{\mathrm{u}, \mathrm{i}}^{2}}{\mathrm{C}_{0} \varepsilon}$

$\mathrm{C}_{0}$ is the Lagrangian Kolmogorov constant. It depends on the Reynolds number and usually varies between 2 and 6 .

In order to integrate (8), (Thomson, 1987) imposes the components of $(\underline{\mathrm{dU}})$ to be Markovian processes, composed by a deterministic term, proportional to $\mathrm{dt}$ and a stochastic term, proportional to a Wiener process $\left(\mathrm{d} \xi_{\mathrm{i}}\right)$. This is defined as a stochastic variable with a Gaussian pdf, a null mean and variance dt (Einstein's notation is valid hereafter for the subscript “ ${ }^{j}$ ”) Equation 12 :

$\mathrm{dU}_{\mathrm{i}}=\mathrm{a}_{\mathrm{i}} \mathrm{dt}+\mathrm{b}_{\mathrm{ij}} \mathrm{d} \xi_{\mathrm{j}}$

Kolmogorov theory provides an estimation of the correlation of the velocity increments, for the time increment $\Delta \mathrm{t}$ tending to zero. Computing the same quantity, under the definition of (12), (Thomson, 1987) finds an expression for $b_{i j}$ Equation 13:

$$
\begin{aligned}
& \overline{\left[\mathrm{U}_{\mathrm{i}}(\mathrm{t}+\Delta \mathrm{t})-\mathrm{U}_{\mathrm{i}}(\mathrm{t})\right]\left[\mathrm{U}_{\mathrm{j}}(\mathrm{t}+\Delta \mathrm{t})-\mathrm{U}_{\mathrm{j}}(\mathrm{t})\right]}= \\
& =\delta_{\mathrm{ij}} \mathrm{C}_{0} \varepsilon \Delta \mathrm{t}, \Delta \mathrm{t} \rightarrow 0 \Rightarrow \mathrm{b}_{\mathrm{ij}}=\delta_{\mathrm{ij}} \sqrt{\mathrm{C}_{0} \varepsilon}
\end{aligned}
$$

In order to derive $\mathrm{a}_{\mathrm{i}}$, (Thomson, 1987) imposes the so-called "well-mixed condition". This affirms that, at every generic time and point and considering a homogeneous distribution of particles all over the domain, the velocity statistics of the Lagrangian (particle) velocity components should be equal to the corresponding Eulerian statistics of the main flow.

This condition guarantees that the simulated particles are consistent with the velocity field of the main flow.

Under the hypothesis of Gaussian pdfs for the Eulerian velocities Equation 14:

$$
\operatorname{pdf}\left(u_{i}\right)=\frac{1}{\sqrt{2 \pi} \sigma_{u, i}} e^{-\frac{1}{2}\left(\frac{u_{i}}{\sigma_{u, i}}\right)^{2}}
$$

(Thomson, 1987) obtains the following 3D non stationary expression Equation 15: 


$$
\left.\mathrm{dU}_{\mathrm{i}}=\left\{\begin{array}{l}
-\frac{\mathrm{C}_{0} \varepsilon}{2}\left(\boldsymbol{V}^{-1}\right)_{\mathrm{ik}} \mathrm{U}_{\mathrm{k}}^{\prime}+\frac{1}{2} \frac{\partial \mathrm{V}_{\mathrm{il}}}{\partial \mathrm{x}_{1}} \\
+\frac{\partial \overline{\mathrm{u}_{\mathrm{i}}}}{\partial \mathrm{t}}+\overline{\mathrm{u}_{1}} \frac{\partial \overline{\mathrm{u}_{\mathrm{i}}}}{\partial \mathrm{x}_{1}} \\
{\left[\begin{array}{l}
\frac{1}{2}\left(\boldsymbol{V}^{-1}\right)_{\mathrm{lj}}\left(\frac{\partial \mathrm{V}_{\mathrm{il}}}{\partial \mathrm{t}}+\overline{\mathrm{u}_{\mathrm{m}}} \frac{\partial \mathrm{V}_{\mathrm{il}}}{\partial \mathrm{x}_{\mathrm{m}}}\right) \\
+\frac{\partial \overline{\mathrm{u}_{\mathrm{i}}}}{\partial \mathrm{x}_{\mathrm{j}}}
\end{array}\right.} \\
\mathrm{U}_{\mathrm{j}}^{\prime}+\frac{1}{2}\left(\boldsymbol{V}^{-1}\right)_{\mathrm{lj}} \frac{\partial \mathrm{V}_{\mathrm{il}}}{\partial \mathrm{x}_{\mathrm{k}}} \mathrm{U}_{\mathrm{j}}^{\prime} \mathrm{U}_{\mathrm{k}}^{\prime}
\end{array}\right]\right\} \mathrm{dt}+\sqrt{\mathrm{C}_{0} \varepsilon} \mathrm{d} \xi_{\mathrm{i}}
$$

where, $\mathrm{V}_{\mathrm{ij}}$ is the covariance matrix of the Eulerian velocity and $\left(\mathrm{V}^{-1}\right)_{\mathrm{lj}}$ is the corresponding inverse matrix.

The general formulation (15) does not seem to have been yet used in micromixing modelling. Several authors (Table 2) use some simplifying formulations (Table 2), as reported in the following sub-sections.

\subsection{Thomson (1987) Scheme: Stationary Regime and Diagonal Covariance Matrix}

In (16) we assume that $(\mathrm{x}, \mathrm{y}, \mathrm{z})=\left(\mathrm{x}_{1}, \mathrm{x}_{2}, \mathrm{x}_{3}\right),(\mathrm{U}, \mathrm{V}$, $\mathrm{W})=\left(\mathrm{U}_{1}, \mathrm{U}_{2}, \mathrm{U}_{3}\right)$ and analogously for the Eulerian velocity components Equation 16:

$$
\begin{aligned}
& \mathrm{dU}=\left[\begin{array}{l}
-\frac{\mathrm{U}^{\prime}}{\mathrm{T}_{\mathrm{Lx}}}+\frac{1}{2} \frac{\partial \sigma_{\mathrm{u}}^{2}}{\partial \mathrm{x}}+\frac{\mathrm{U}^{\prime}}{2 \sigma_{\mathrm{u}}^{2}} \\
\left(\mathrm{U} \frac{\partial \sigma_{\mathrm{u}}^{2}}{\partial \mathrm{x}}+\mathrm{V} \frac{\partial \sigma_{\mathrm{u}}^{2}}{\partial \mathrm{y}}+\mathrm{W} \frac{\partial \sigma_{\mathrm{u}}^{2}}{\partial \mathrm{z}}\right) \\
+\mathrm{U} \frac{\partial \overline{\mathrm{u}}}{\partial \mathrm{x}}+\mathrm{V} \frac{\partial \overline{\mathrm{u}}}{\partial \mathrm{y}}+\mathrm{W} \frac{\partial \overline{\mathrm{u}}}{\partial \mathrm{z}}
\end{array}\right] \mathrm{dt}+\sqrt{\mathrm{C}_{0} \varepsilon} \mathrm{d} \xi_{\mathrm{u}} \\
& \mathrm{dV}=\left[\begin{array}{l}
\left(\begin{array}{l}
\mathrm{T}_{\mathrm{Ly}} \\
\left(\frac{\partial \sigma_{\mathrm{v}}^{2}}{\partial \mathrm{x}}+\mathrm{V} \frac{\partial \sigma_{\mathrm{v}}^{2}}{\partial \mathrm{y}}+\mathrm{W} \frac{\partial \sigma_{\mathrm{v}}^{2}}{\partial \mathrm{z}}\right)
\end{array}\right] \mathrm{Vt}+\sqrt{\mathrm{C}_{0} \varepsilon} \mathrm{d} \xi_{\mathrm{v}} \\
+\mathrm{U} \frac{\partial \overline{\mathrm{v}}}{\partial \mathrm{x}}+\mathrm{V} \frac{\partial \overline{\mathrm{v}}}{\partial \mathrm{y}}+\mathrm{W} \frac{\partial \overline{\mathrm{v}}}{\partial \mathrm{z}}
\end{array}\right] \\
& \mathrm{dW}=\left[\begin{array}{l}
\mathrm{W} \\
-\frac{\mathrm{W}}{\mathrm{T}_{\mathrm{Lz}}}+\frac{1}{2} \frac{\partial \sigma_{\mathrm{w}}^{2}}{\partial \mathrm{z}}+\frac{\mathrm{W}}{2 \sigma_{\mathrm{w}}^{2}} \\
\left(\mathrm{U} \frac{\partial \sigma_{\mathrm{w}}^{2}}{\partial \mathrm{x}}+\mathrm{V} \frac{\partial \sigma_{\mathrm{w}}^{2}}{\partial \mathrm{y}}+\mathrm{W} \frac{\partial \sigma_{\mathrm{w}}^{2}}{\partial \mathrm{z}}\right) \\
+\mathrm{U} \frac{\partial \overline{\mathrm{w}}}{\partial \mathrm{x}}+\mathrm{V} \frac{\partial \overline{\mathrm{w}}}{\partial \mathrm{y}}+\mathrm{W} \frac{\partial \overline{\mathrm{w}}}{\partial \mathrm{z}}
\end{array}\right] \mathrm{dt}+\sqrt{\mathrm{C}_{0} \varepsilon} \mathrm{d} \xi_{\mathrm{w}}
\end{aligned}
$$

\subsection{Thomson (1987) Scheme: Grid Turbulence- Like Solution}

With a scalar mean flow Taylor translation hypothesis can be assumed, so that: $\mathrm{X}=\mathrm{Ut}$ and the macromixing scheme reduces to 1D (even assuming transversal homogeneity) Equation 17:

$$
\begin{aligned}
& \mathrm{dW}=\left[-\frac{\mathrm{W}}{\mathrm{T}_{\mathrm{L}}}+\frac{\mathrm{W} \overline{\mathrm{U}}}{2 \sigma_{\mathrm{w}}^{2}} \frac{\partial \sigma_{\mathrm{w}}^{2}}{\partial \mathrm{x}}\right] \mathrm{dt} \\
& +\sqrt{\mathrm{C}_{0} \varepsilon} \mathrm{d} \xi_{\mathrm{W}}=\left[-\frac{\mathrm{W}}{\mathrm{T}_{\mathrm{L}}}+\frac{\mathrm{W}}{2 \sigma_{\mathrm{w}}^{2}} \frac{\mathrm{d} \sigma_{\mathrm{w}}^{2}}{\mathrm{dt}}\right] \mathrm{dt} \\
& +\sqrt{\mathrm{C}_{0} \varepsilon} \mathrm{d} \xi_{\mathrm{W}}, \quad \overline{\mathrm{U}}=\frac{\mathrm{dX}}{\mathrm{dt}}
\end{aligned}
$$

2.4. Thomson (1987) Scheme: Stationary Regime, Diagonal Covariance Matrix, 2D Dispersion, Mean Velocity Aligned with Z-Axis, Horizontal Homogeneity

Under these conditions Taylor translation hypothesis can be applied and the macromixing scheme reduces 2D Equation 18:

$$
\begin{aligned}
& \mathrm{dV}=\left[\mathrm{W} \frac{\partial \sigma_{\mathrm{v}}^{2}}{\partial \mathrm{z}}-\mathrm{C}_{0} \varepsilon\right] \frac{\mathrm{V}}{2 \sigma_{\mathrm{v}}^{2}} \mathrm{dt}+\sqrt{\mathrm{C}_{0} \varepsilon} \mathrm{d} \xi_{\mathrm{v}} \\
& \mathrm{dW}=\left[-\frac{\mathrm{W}}{\mathrm{T}_{\mathrm{L}}}+\frac{1}{2 \sigma_{\mathrm{w}}^{2}} \cdot \frac{\partial \sigma_{\mathrm{w}}^{2}}{\partial \mathrm{z}}\left(\sigma_{\mathrm{w}}^{2}+\mathrm{W}^{2}\right)\right] \mathrm{dt}+\sqrt{\mathrm{C}_{0} \varepsilon} \mathrm{d} \xi_{\mathrm{w}}
\end{aligned}
$$

\subsection{Thomson (1987) Scheme: Solution for Fluctuating Velocities}

Thomson scheme can be reformulated splitting the instantaneous velocity components, according to Reynolds' decomposition and considering only the fluctuating part of velocity as a Markovian process Equation 19:

$$
\begin{aligned}
& d U_{i}^{\prime}=a_{i}^{\prime} d t+b_{i j} d \xi_{i} \\
& U_{i}(t+d t)=\overline{u_{i}}(t+d t)+U_{i}^{\prime}(t)+d U_{i}^{\prime}(t)
\end{aligned}
$$

Under the hypothesis underlying (16) the corresponding solution for the fluctuating velocity increments is Equation 20: 
Andrea Amicarelli et al. / American Journal of Environmental Science 8 (6) (2012) 577-590

Table 2. A schematic overview of some Lagrangian micromixing studies

\begin{tabular}{|c|c|c|c|c|c|c|c|c|c|}
\hline Year & Authors & $\begin{array}{l}\text { Boundary Layer } \\
\text { BL (model } \\
\text { dimensionality) }\end{array}$ & $\begin{array}{l}\text { Macromixing } \\
\text { equation } \\
\left(\mathrm{C}_{0} \text { value }\right)\end{array}$ & $\begin{array}{l}\text { Micromixing } \\
\text { scheme }\end{array}$ & $\begin{array}{l}t_{\mathrm{m}} \\
\text { formulation } \\
\left(\mu \text { or } b, C_{\square}\right)\end{array}$ & $\begin{array}{l}\mathrm{i}_{\mathrm{C}} \\
\min \\
\max \end{array}$ & $\begin{array}{l}\mathrm{S}_{\mathrm{C}} \\
\min \\
\max \end{array}$ & $\begin{array}{l}\mathrm{K}_{\mathrm{C}} \\
\min \\
\max \end{array}$ & $\begin{array}{l}\mathrm{C}_{99} / \overline{\mathrm{C}} \\
\min \\
\max \end{array}$ \\
\hline 2004a & Sawford (2004a) & $\begin{array}{l}\text { grid turbulence } \\
\text { (1D) }\end{array}$ & $\begin{array}{l}(2.17) \\
(2.07-4.89)\end{array}$ & $\begin{array}{l}\text { IECM, IEM, } \\
\text { VCIEM }\end{array}$ & $\begin{array}{l}\text { Sawford } \\
2004 \mathrm{a} \\
(1.2, /)\end{array}$ & $\begin{array}{l}0 \\
1.2\end{array}$ & n.a. & n.a. & n.a. \\
\hline $2004 b$ & Sawford (2004b) & $\begin{array}{l}\text { grid turbulence } \\
\text { (1D) }\end{array}$ & $(2.17)(3)$ & IECM, IEM & $\begin{array}{l}\text { Sawford } 2004 \mathrm{a} \\
(1.2,1.81-3)\end{array}$ & $\begin{array}{l}0.2 \\
1.2\end{array}$ & $\begin{array}{l}-1.5 \\
2.8\end{array}$ & $\begin{array}{l}1 \\
4.5\end{array}$ & $\begin{array}{l}1.6 \\
2.5\end{array}$ \\
\hline $2005 a$ & $\begin{array}{l}\text { Cassiani et al. } \\
\text { (2005a) }\end{array}$ & $\begin{array}{l}\text { homogeneous } \\
\text { turbulence + NBL } \\
\text { wind tunnel (1D) }\end{array}$ & $(2.18)-1 \mathrm{D}(6)$ & IEM & $\begin{array}{l}\text { Cassiani et al., } \\
2005 \text { a }(0.65,2)\end{array}$ & $\begin{array}{l}0 \\
4.5\end{array}$ & n.a. & n.a. & n.a. \\
\hline $2005 b$ & $\begin{array}{l}\text { Cassiani et al. } \\
(2005 b)\end{array}$ & $\begin{array}{l}\text { Convective BL } \\
\text { (2D) }\end{array}$ & $(2.24)$ & IECM & $\begin{array}{l}\text { Cassiani et al. } \\
(2005 \mathrm{a}) \\
(0.7-0.8,2)\end{array}$ & $\begin{array}{l}0.5 \\
4\end{array}$ & n.a. & n.a. & n.a. \\
\hline $2005 \mathrm{c}$ & $\begin{array}{l}\text { Cassiani et al. } \\
(2005 \mathrm{c})\end{array}$ & $\begin{array}{l}\text { NBL with } \\
\text { canopy (2D) }\end{array}$ & $\begin{array}{l}(2.23) \\
(2)\end{array}$ & IECM & $\begin{array}{l}\text { Pope (2000) } \\
(/, 2)\end{array}$ & n.a. & n.a. & n.a. & n.a. \\
\hline $2005 a$ & $\begin{array}{l}\text { Luhar and Sawford } \\
\text { (2005a) }\end{array}$ & $\begin{array}{l}\text { CBL, } 2 \\
\text { sources(2D) }\end{array}$ & $\begin{array}{l}(2.24) \\
(3)\end{array}$ & IECM & $\begin{array}{l}\text { Sawford 2004a } \\
(1.1, /)\end{array}$ & n.a. & $\begin{array}{l}0.4 \\
2.1\end{array}$ & $\begin{array}{l}3.5 \\
9\end{array}$ & n.a. \\
\hline $2005 \mathrm{~b}$ & $\begin{array}{l}\text { Luhar and Sawford } \\
\text { (2005b) }\end{array}$ & CBL (2D) & $\begin{array}{l}(2.24) \\
(3)\end{array}$ & IECM & $\begin{array}{l}\text { Sawford } 2004 \mathrm{a} \\
(0.9 \text { for line, } \\
0.6 \text { for point, } /)\end{array}$ & $\begin{array}{l}0 \\
10\end{array}$ & $\begin{array}{l}3 \\
15\end{array}$ & $\begin{array}{l}18 \\
100\end{array}$ & n.a. \\
\hline $2006 \mathrm{a}$ & Sawford (2006a) & $\begin{array}{l}\text { grid turbulence, } \\
2 \text { sources (1D) }\end{array}$ & $\begin{array}{l}(2.17) \\
(3)\end{array}$ & $\begin{array}{l}\text { IECM (mixture } \\
\text { fraction scheme } \\
\text { for reactions) }\end{array}$ & $\begin{array}{l}\text { Sawford 2004a } \\
(0.5, /)\end{array}$ & n.a. & n.a. & n.a. & n.a. \\
\hline $2006 b$ & Sawford (2006b) & $\begin{array}{l}\text { grid turbulence } \\
\text { (1D) }\end{array}$ & $\begin{array}{l}(2.17) \\
(1.4)\end{array}$ & IECM & $\begin{array}{l}\text { Sawford 2004a } \\
(0.5, /)\end{array}$ & $\begin{array}{l}0 \\
1\end{array}$ & $\begin{array}{l}-80 \\
80\end{array}$ & $\begin{array}{l}100 \\
104\end{array}$ & $\begin{array}{l}1 \\
100\end{array}$ \\
\hline $2007 \mathrm{a}$ & $\begin{array}{l}\text { Cassiani et al. } \\
\text { (2007a) }\end{array}$ & $\begin{array}{l}\text { NBL with } \\
\text { canopy (2D) } \\
\text { Multiple sources }\end{array}$ & $\begin{array}{l}(2.23) \\
(2)\end{array}$ & IECM & $\begin{array}{l}\text { Cassiani et al., } \\
(2005 \mathrm{a})(0.82,2)\end{array}$ & n.a. & $\begin{array}{l}-0.5 \\
4\end{array}$ & $\begin{array}{l}2 \\
15\end{array}$ & n.a. \\
\hline $2007 b$ & $\begin{array}{l}\text { Cassiani et al. } \\
\text { (2007b) }\end{array}$ & $\begin{array}{l}\text { NBL with } \\
\text { canopy (2D) }\end{array}$ & $\begin{array}{l}(2.23) \\
(2)\end{array}$ & IECM, IEM & $\begin{array}{l}\text { Cassiani et al., } \\
(2005 \mathrm{a})(0.8,2)\end{array}$ & n.a. & n.a. & n.a. & n.a. \\
\hline 2007 & $\begin{array}{l}\text { Dixon and Tomlin } \\
\text { (2007) }\end{array}$ & $\begin{array}{l}\text { Multiple sources } \\
\text { wind tunnel NBL } \\
+ \text { lid-driven } \\
\text { cavity (2D) }\end{array}$ & $\begin{array}{l}\text { n.a. } \\
(5)\end{array}$ & IEM & Pope (2000) & $\begin{array}{l}0 \\
(/, 2)\end{array}$ & n.a. & $\begin{array}{l}\text { n.a. } \\
8\end{array}$ & $7^{1.5}$ \\
\hline $2011 \mathrm{a}$ & $\begin{array}{l}\text { Amicarelli et al. } \\
\text { (2011a) }\end{array}$ & $\begin{array}{l}\text { wind tunnel } \\
\text { NBL 2D }\end{array}$ & $\begin{array}{l}(2.18) \\
(5)\end{array}$ & IECM, IEM & $\begin{array}{l}\text { Cassiani et al., } \\
\text { 2005a }(0.65,2)\end{array}$ & $\begin{array}{l}0 \\
2.5\end{array}$ & n.a. & n.a. & n.a. \\
\hline $2011 b$ & $\begin{array}{l}\text { Amicarelli et al. } \\
\text { (2011b) }\end{array}$ & $\begin{array}{l}\text { wind tunnel } \\
\text { NBL, canopy } \\
\text { (3D) }\end{array}$ & $\begin{array}{l}(2.16) \\
(2)\end{array}$ & IECM & $\begin{array}{l}\text { Amicarelli et al. } \\
(2011 b)(0.65,2)\end{array}$ & $\begin{array}{l}10^{-1} \\
10^{2}\end{array}$ & n.a. & n.a. & n.a. \\
\hline $2011 \mathrm{a}$ & $\begin{array}{l}\text { Postma et al. } \\
\text { (2011a) }\end{array}$ & $\begin{array}{l}\text { wind tunnel } \\
\text { NBL (2D) }\end{array}$ & $\begin{array}{l}(2.18) \\
(6)\end{array}$ & IECM & $\begin{array}{l}\text { Cassiani et al., } \\
\text { 2005a }(0.75,2)\end{array}$ & $\begin{array}{l}1 \\
3.5\end{array}$ & n.a. & n.a. & n.a. \\
\hline $2011 b$ & $\begin{array}{l}\text { Postma et al. } \\
\text { (2011b) }\end{array}$ & $\begin{array}{l}\text { NBL with } \\
\text { canopy (3D) } \\
\text { multiple sources }\end{array}$ & $\begin{array}{l}(2.15)- \\
\text { statio. (2) }\end{array}$ & IECM & $\begin{array}{l}\text { Cassiani et al., } \\
(2005 \mathrm{a})(0.82,2)\end{array}$ & $\begin{array}{l}0.4 \\
1.1\end{array}$ & n.a. & n.a. & n.a. \\
\hline 2012 & $\begin{array}{l}\text { Leuzzi et al. } \\
\text { (2012) }\end{array}$ & $\begin{array}{l}\text { wind tunnel } \\
\text { NBL, canopy } \\
\text { (3D) }\end{array}$ & $\begin{array}{l}(2.16) \\
(2)\end{array}$ & IECM, IEM & $\begin{array}{l}\text { Amicarelli et al. } \\
(2011 b)(0.33,2)\end{array}$ & $\begin{array}{l}0.2 \\
1000\end{array}$ & $\begin{array}{l}0 \\
24\end{array}$ & n.a. & n.a. \\
\hline in press & $\begin{array}{l}\text { Amicarelli et al. } \\
\text { (2012) }\end{array}$ & $\begin{array}{l}\text { wind tunnel } \\
\text { BL, canopy(2D) }\end{array}$ & $\begin{array}{l}(2.16)-2 \mathrm{D} \\
(2)\end{array}$ & IECM & $\begin{array}{l}\text { Amicarelli et al. } \\
(2011 b)(0.65,2)\end{array}$ & $\begin{array}{l}10^{-1} \\
100\end{array}$ & n.a. & n.a. & n.a. \\
\hline
\end{tabular}


$\mathrm{dU^{ \prime }}=\left[\begin{array}{l}-\frac{\mathrm{U}^{\prime}}{\mathrm{T}_{\mathrm{Lx}}}+\frac{1}{2} \frac{\partial \sigma_{\mathrm{u}}^{2}}{\partial \mathrm{x}}+\frac{\mathrm{U}^{\prime}}{2 \sigma_{\mathrm{u}}^{2}} \\ \left(\mathrm{U}^{\prime} \frac{\partial \sigma_{\mathrm{u}}^{2}}{\partial \mathrm{x}}+\mathrm{V}^{\prime} \frac{\partial \sigma_{\mathrm{u}}^{2}}{\partial \mathrm{y}}+\mathrm{W}^{\prime} \frac{\partial \sigma_{\mathrm{u}}^{2}}{\partial \mathrm{z}}\right)\end{array}\right] \mathrm{dt}+\sqrt{\mathrm{C}_{0} \varepsilon} \mathrm{d} \xi_{\mathrm{u}}$
$\mathrm{d} \mathrm{V}^{\prime}=\left[\begin{array}{l}-\frac{\mathrm{V}^{\prime}}{\mathrm{T}_{\mathrm{Ly}}}+\frac{1}{2} \frac{\partial \sigma_{\mathrm{v}}^{2}}{\partial \mathrm{y}}+\frac{\mathrm{V}^{\prime}}{2 \sigma_{\mathrm{v}}^{2}} \\ \left(\mathrm{U}^{\prime} \frac{\partial \sigma_{\mathrm{v}}^{2}}{\partial \mathrm{x}}+\mathrm{V}^{\prime} \frac{\partial \sigma_{\mathrm{v}}^{2}}{\partial \mathrm{y}}+\mathrm{W}^{\prime} \frac{\partial \sigma_{\mathrm{v}}^{2}}{\partial \mathrm{z}}\right)\end{array}\right] \mathrm{dt}+\sqrt{\mathrm{C}_{0} \varepsilon} \mathrm{d} \xi_{\mathrm{v}}$
$\mathrm{dW} \mathrm{W}^{\prime}=\left[\begin{array}{l}-\frac{\mathrm{W}^{\prime}}{\mathrm{T}_{\mathrm{Lz}}}+\frac{1}{2} \frac{\partial \sigma_{\mathrm{w}}^{2}}{\partial \mathrm{z}}+\frac{\mathrm{W}^{\prime}}{2 \sigma_{\mathrm{w}}^{2}} \\ \left(\mathrm{U}^{\prime} \frac{\partial \sigma_{\mathrm{w}}^{2}}{\partial \mathrm{x}}+\mathrm{V}^{\prime} \frac{\partial \sigma_{\mathrm{w}}^{2}}{\partial \mathrm{y}}+\mathrm{W}^{\prime} \frac{\partial \sigma_{\mathrm{w}}^{2}}{\partial \mathrm{z}}\right)\end{array}\right] \mathrm{dt}+\sqrt{\mathrm{C}_{0} \varepsilon} \mathrm{d} \xi_{\mathrm{w}}$

\subsection{Cassiani et al. (2007a): A Scheme for Non- Diagonal Covariance Matrix in Surface Boundary Layers}

The well-mixed condition can be fulfilled, even assuming other velocity pdfs. The generic form of the solution is Equation 21:

$$
\begin{aligned}
& d U_{i}=a_{i} d t+b d \xi_{i}=-\frac{1}{2} C_{0} \varepsilon U_{i} d t \\
& +\left(\alpha_{i}+\beta_{i j} U_{j}+\gamma_{i j k} U_{j} U_{k}\right) d t+\sqrt{C_{0}} \varepsilon d \xi_{i}
\end{aligned}
$$

(Cassiani et al., 2007a) assume correlated Gaussian pdfs for the velocity components (surface BLs, as for canopy turbulence; 2D) Equation 22:

$$
\operatorname{pdf}(\mathrm{u}, \mathrm{w})=\frac{1}{2 \pi \sqrt{\sigma_{\mathrm{u}}^{2} \sigma_{\mathrm{w}}^{2}-\sigma_{\mathrm{uw}}^{2}}} \cdot \exp \left(-\frac{\mathrm{u}^{2} \sigma_{\mathrm{w}}^{2}+\mathrm{w}^{2} \sigma_{\mathrm{u}}^{2}-2 \mathrm{uw} \sigma_{\mathrm{uw}}^{2}}{2\left(\sigma_{\mathrm{u}}^{2} \sigma_{\mathrm{w}}^{2}-\sigma_{\mathrm{uw}}^{2}\right)}\right)
$$

The solution (20) is then modified (the vertical gradients of the velocity statistics are considered much larger of the corresponding horizontal components: horizontal homogeneity, (Cassiani et al., 2007a) Equation 23:

$$
\mathrm{dU}^{\prime}=\left\{\begin{array}{c}
-\frac{1}{2 \sigma_{\mathrm{u}}^{2} \sigma_{\mathrm{w}}^{2}-\sigma_{\mathrm{uw}}^{2}} \mathrm{C}_{0} \varepsilon\left(\sigma_{\mathrm{w}}^{2} \mathrm{U}^{\prime}-\sigma_{\mathrm{uw}}^{2} \mathrm{~W}^{\prime}\right)+ \\
\frac{1}{2} \frac{\partial \sigma_{\mathrm{uw}}^{2}}{\partial \mathrm{z}}+\left(\frac{1}{2 \sigma_{\mathrm{u}}^{2} \sigma_{\mathrm{w}}^{2}-\sigma_{\mathrm{uw}}^{2}}\right) \\
{\left[\begin{array}{l}
\mathrm{U}^{\prime} \mathrm{W}^{\prime}\left(\sigma_{\mathrm{w}}^{2} \frac{\partial \sigma_{\mathrm{u}}^{2}}{\partial \mathrm{z}}-\sigma_{\mathrm{uw}}^{2} \frac{\partial \sigma_{\mathrm{uw}}^{2}}{\partial \mathrm{z}}\right) \\
+\left(\mathrm{W}^{\prime}\right)^{2}\left(\sigma_{\mathrm{u}}^{2} \frac{\partial \sigma_{\mathrm{uw}}^{2}}{\partial \mathrm{z}}-\sigma_{\mathrm{uw}}^{2} \frac{\partial \sigma_{\mathrm{u}}^{2}}{\partial z}\right)
\end{array}\right.}
\end{array}\right\} \mathrm{dt}+\sqrt{\mathrm{C}_{0} \varepsilon} \mathrm{d} \xi_{\mathrm{u}}
$$

$$
\begin{gathered}
d^{\prime}=\left[-\frac{V^{\prime}}{T_{L y}}+\frac{V^{\prime} W^{\prime}}{2 \sigma_{v}^{2}} \frac{\partial \sigma_{v}^{2}}{\partial z}\right] d t+\sqrt{C_{0} \varepsilon} d \xi_{v} \\
d W^{\prime}=\left\{\begin{array}{l}
2 \frac{1}{2 \sigma_{u}^{2} \sigma_{w}^{2}-\sigma_{u w}^{2}} C_{0} \varepsilon\left(\sigma_{u}^{2} W^{\prime}-\sigma_{u w}^{2} U^{\prime}\right) \\
+\frac{1}{2} \frac{\partial \sigma_{w}^{2}}{\partial z}+\left(\frac{1}{2 \sigma_{u}^{2} \sigma_{w}^{2}-\sigma_{u w}^{2}}\right) \\
{\left[\begin{array}{l}
U^{\prime} W^{\prime}\left(\sigma_{w}^{2} \frac{\partial \sigma_{u w}^{2}}{\partial z}-\sigma_{u w}^{2} \frac{\partial \sigma_{w}^{2}}{\partial z}\right) \\
\left(W^{\prime}\right)^{2}\left(\sigma_{u}^{2} \frac{\partial \sigma_{w}^{2}}{\partial z}-\sigma_{u w}^{2} \frac{\partial \sigma_{u w}^{2}}{\partial z}\right)
\end{array}\right] d t+\sqrt{C_{0} \varepsilon} d \xi_{u}}
\end{array}\right\}
\end{gathered}
$$

\subsection{Luhar and Britter (1989): Simplified 2D Macromixing in Convective Boundary Layers}

Convective boundary layers are characterized by nonGaussian pdfs of the vertical component of velocity. Luhar and Britter (1989) derive a macromixing solution, according to the procedure described in (Thomson, 1987), assuming homogeneous conditions for the horizontal standard deviation of velocity. Further the probability density function of the $2 \mathrm{D}$ velocity particle component is a product of 2 conditioned pdfs, one per velocity component. The first (horizontal) is a Gaussian function, whilst the vertical is a sum of two Gaussian functions, modelling the updraft and downdraft velocities. The solution (20) is then modified, even assuming horizontal homogeneity $\left(\mathrm{a}_{\mathrm{LB}}\right.$ is described in detail in (Luhar and Sawford, 2005b) Equation 24:

$$
\begin{aligned}
& \mathrm{dV}^{\prime}=-\frac{\mathrm{V}^{\prime}}{\mathrm{T}_{\mathrm{L}}} \mathrm{dt}+\sqrt{\mathrm{C}_{0} \varepsilon} d \xi_{\mathrm{v}} \\
& \mathrm{dW}^{\prime}=\mathrm{a}_{\mathrm{LB}} \mathrm{dt}+\sqrt{\mathrm{C}_{0} \varepsilon} \mathrm{d}_{\mathrm{w}}
\end{aligned}
$$

\subsection{Micromixing Schemes}

During the micromixing process, particle concentrations evolve because of molecular diffusion phenomena and physical or chemical transformations (reactive pollutants). The two more common micromixing schemes are summarized in the following sub-sections, which even provide references for the formulations of the mixing time scale $t_{m}$ and $a$ micromixing formulation for reactive pollutants. Algorithm simplifications are finally presented. 


\subsection{IECM Micromixing Scheme}

The most common micromixing scheme is the Interaction by Exchange with the Conditional Mean (IECM; (Pope, 1998; Sawford, 2004a) for passive pollutants) Equation 25:

$$
\frac{\mathrm{dC}}{\mathrm{dt}}=-\frac{\mathrm{C}-\langle\mathrm{C} \mid \underline{\mathrm{U}}\rangle}{\mathrm{t}_{\mathrm{m}}}
$$

Particles exchange pollutant mass with the surrounding environment depending on the difference between their particle (instantaneous) concentration and the conditional mean (fixed a point and time). Considering that a fluid particle should exchange pollutant mass only with fluid particles belonging to the same realization, the parameter $\langle\underline{C} \mid \underline{U}\rangle$ is someway representative of an instantaneous plume, as only the particles with the same velocity in the on-going computational point and time are involved in the estimation of the conditional mean.

The diffusive exchanges are ruled by the mixing time scale $t_{m}$. Its formulation is analysed. When $t_{m}$ tends to infinity particles behave as conservative elements (macromixing limit). On the other hand, when $t_{m}$ tends to zero, particle concentration tends to the conditional mean: the concentration fluctuations are then underestimated and only caused by the plume meandering phenomena, ruled by the largest turbulent vortices. Integrating (2.25) in time we obtain the particle concentration at the end of the time step, under the hypothesis that the conditional mean is constant during the time step. This implies that $\Delta \mathrm{t}<<\mathrm{t}_{\mathrm{m}}$ this scheme satisfies the conditions (a), (b) and (d), as reported at the beginning. The particle concentration exponentially decreases towards the conditional mean during the micromixing process. IECM then reduces the concentration fluctuations which would be estimated by a macromixing model. Further all the possible values of the particle concentrations lye between zero and the source concentration. Finally it can be demonstrated that the mean is unaffected by the IECM scheme (Sawford, 2004a).

Replacing the Lagrangian derivative of concentration in (7), $\varepsilon_{\mathrm{C}}$ can be expressed as follows Equation 26:

$$
\begin{aligned}
& \varepsilon_{\mathrm{C}}(\mathrm{IECM})=-2 \mathrm{C}^{\prime}\left(\frac{\mathrm{C}-\langle\mathrm{C} \mid \underline{\mathrm{U}}\rangle}{\mathrm{t}_{\mathrm{m}}}\right)=-2 \overline{\left(\frac{\mathrm{C}^{\prime} \overline{\mathrm{C}}}{\mathrm{t}_{\mathrm{m}}}+\frac{\sigma_{\mathrm{C}}^{2}}{\mathrm{t}_{\mathrm{m}}}-\frac{\mathrm{C}^{\prime}\langle\mathrm{C} \mid \underline{\mathrm{U}}\rangle}{\mathrm{t}_{\mathrm{m}}}\right)} \Rightarrow \\
& \Rightarrow \varepsilon_{\mathrm{C}}=-\frac{2}{\mathrm{t}_{\mathrm{m}}}\left[\sigma_{\mathrm{C}}^{2}-\overline{\left(\mathrm{C}^{\prime}\langle\mathrm{C} \mid \underline{\mathrm{U}}\rangle\right)}\right]
\end{aligned}
$$

\subsection{IEM Micromixing Scheme}

A simpler micromixing scheme is represented by the IEM model (Interaction by Exchange with the Mean; Villermaux and Devillon (1972) and Dopazo and O’Brien (1974) Equation 27:

$\frac{\mathrm{dC}}{\mathrm{dt}}=-\frac{\mathrm{C}-\overline{\mathrm{C}}}{\mathrm{t}_{\mathrm{m}}}$

The conditional mean of the IECM equation is here replaced by the concentration mean, with a reduced computational cost during the macromixing phase, as IEM is equivalent to a degenerated IECM, with only one velocity class (requiring fewer particles to provide robust statistics for the means). IEM is theoretically less precise than IECM: Particles exchange pollutant mass even depending on the concentration of particles which belong to very different realizations (non-physical behaviour). Further (Sawford, 2004a) demonstrates that IEM scheme alters the balance equation of the mean and its turbulent flux. At the same time it is immediate to demonstrate that (3) is altered by IEM Equation 28:

$\left\langle\frac{\mathrm{dC}}{\mathrm{dt}} \mid \underline{\mathrm{U}}\right\rangle=-\frac{\langle\mathrm{C} \mid \underline{\mathrm{U}}\rangle-\overline{\mathrm{C}}}{\mathrm{t}_{\mathrm{m}}} \neq 0$

The dissipation of the concentration variance can be expressed according to the IEM scheme Equation 29:

$$
\begin{aligned}
& \varepsilon_{\mathrm{C}}(\text { IEM })=-2 \overline{\mathrm{C}^{\prime}\left(\frac{\mathrm{C}-\overline{\mathrm{C}}}{\mathrm{t}_{\mathrm{m}}}\right)}=-2 \overline{\left(\frac{\mathrm{C}^{\prime} \overline{\mathrm{C}}}{\mathrm{t}_{\mathrm{m}}}+\frac{\sigma_{\mathrm{C}}^{2}}{\mathrm{t}_{\mathrm{m}}}-\frac{\mathrm{C}^{\prime} \overline{\mathrm{C}}}{\mathrm{t}_{\mathrm{m}}}\right)} \Rightarrow \\
& \Rightarrow \varepsilon_{\mathrm{C}}=-2 \frac{\sigma_{\mathrm{C}}^{2}}{\mathrm{t}_{\mathrm{m}}}
\end{aligned}
$$

We intuitively notice that, averagely, the instantaneous concentration is closer to the conditional mean than to the mean concentration (but when $\langle\mathrm{C} \mid \underline{\mathrm{U}}\rangle$ and $\overline{\mathrm{C}}$ are equal then IECM and IEM provide the same results). Comparing (26) and (29) it can be shown that IEM overestimates the dissipation of the concentration variance with respect to the IECM scheme. A quantitative and brief demonstration of this statement is provided in the following, developing the term $\left(\overline{\mathrm{C}^{\prime}\langle\mathrm{C} \mid \underline{\mathrm{U}}\rangle}\right)$. First we notice that Equation 30:

$$
\overline{\mathrm{C}^{\prime} \overline{\mathrm{C}}}=0 \Rightarrow \quad \overline{\mathrm{C}^{\prime}\langle\mathrm{C} \mid \underline{\mathrm{U}}\rangle}=\overline{\mathrm{C}^{\prime}(\langle\mathrm{C} \mid \underline{\mathrm{U}}\rangle-\overline{\mathrm{C}})}
$$


Then we consider that the mean of a parameter is equal to the mean of its conditional mean Equation 31:

$$
\begin{aligned}
& \overline{\mathrm{C}^{\prime}\langle\mathrm{C} \mid \underline{\mathrm{U}}\rangle}=\overline{\mathrm{C}^{\prime}(\langle\mathrm{C} \mid \underline{\mathrm{U}}\rangle-\overline{\mathrm{C}})}=\overline{\left\langle\left[\mathrm{C}^{\prime}(\langle\mathrm{C} \mid \underline{\mathrm{U}}\rangle-\overline{\mathrm{C}})\right] \mid \underline{\mathrm{U}}\right\rangle} \\
& =\overline{(\langle\mathrm{C} \mid \underline{\mathrm{U}}\rangle-\overline{\mathrm{C}})\langle\overline{\mathrm{C}} \mid \underline{\mathrm{U}}\rangle}=\overline{(\langle\mathrm{C} \mid \underline{\mathrm{U}}\rangle-\overline{\mathrm{C}})\langle(\mathrm{C}-\overline{\mathrm{C}}) \mid \underline{\mathrm{U}}\rangle} \\
& =\overline{(\langle\mathrm{C} \mid \underline{\mathrm{U}}\rangle-\overline{\mathrm{C}})(\langle\mathrm{C} \mid \underline{\mathrm{U}}\rangle-\overline{\mathrm{C}})}=\overline{(\langle\mathrm{C} \mid \underline{\mathrm{U}}\rangle-\overline{\mathrm{C}})^{2}}>0
\end{aligned}
$$

Considering (31) we can finally deduce that Equation 32:

$$
\overline{\mathrm{C}^{\prime}\langle\mathrm{C} \mid \underline{\mathrm{U}}\rangle} \geq 0 \Rightarrow \quad\left|\varepsilon_{\mathrm{C}}\right|(\mathrm{IEM}) \geq\left|\varepsilon_{\mathrm{C}}\right|(\mathrm{IECM})
$$

Introducing (31) in (26) we even obtain a clearer formulation for $\varepsilon \mathrm{C}$, as modelled by IECM Equation 33:

$$
\varepsilon_{\mathrm{C}}(\mathrm{IECM})=-\frac{2}{\mathrm{t}_{\mathrm{m}}}\left[\sigma_{\mathrm{C}}^{2}-\overline{\left(\mathrm{C}^{\prime}\langle\mathrm{C} \mid \underline{\mathrm{U}}\rangle\right)}\right]=-\frac{2}{\mathrm{t}_{\mathrm{m}}}\left[\sigma_{\mathrm{C}}^{2}-\overline{(\langle\mathrm{C} \mid \underline{\mathrm{U}}\rangle-\overline{\mathrm{C}})^{2}}\right]
$$

As resulting for example from (Amicarelli et al., 2011a; Cassiani et al., 2007b; Leuzzi et al., 2012), IECM is more precise than IEM. The difference in terms of computational costs, does not seem to justify the use of IEM for accurate simulations, but for preliminary simulations, in order to save memory and computational time.

\subsection{The Mixing Time Scale}

The alternative definitions of the time scale of the micromixing processes, or mixing time scale $\left(t_{m}\right)$, share the same principles. The dissipation of the concentration variance is related to molecular diffusion, whose effects are appreciable, even in turbulent regimes, because the gradients of the instantaneous concentrations are large. Increasing the turbulent kinetic energy the molecular diffusion fluxes grow, because particles are continuously in contact with new surrounding particles, with different concentrations. Further only when the plume length scale is comparable with the boundary layer depth all the vortices contribute to the relative dispersion of particles. The Lagrangian derivative of concentration should be then someway related to the difference of the particle concentrations (as resulting from IECM or IEM), to the molecular diffusion coefficient, the kinetic energy, the plume and the source dimensions and the integral Lagrangian time scale. These last parameters are then involved in the formulation of $t_{\mathrm{m}}$.

\subsection{The Mixing Time Scale: Sawford (2004a) Formulation}

Sawford (2004a), shows that in the near-and medium-field $t_{m}$ is substantially linear in $t_{f}$ (the fly time) and depends on the source size $(\mathrm{D}$, related to the plume spread at source $\sigma_{0}$ ) only very close to the emission point Equation 34:

$\mathrm{t}_{\mathrm{m}}=\left(\frac{\sigma_{0}^{2}}{\varepsilon}\right)^{\frac{1}{3}}+\mathrm{bt}_{\mathrm{f}}$

where, $b$ is a proportionality constant between $t_{m}$ and $t_{f}$.

\subsection{The Mixing Time Scale: Cassiani et al. (2005a) Formulation}

Following (Sawford, 2004a; Sykes et al., 1984), Cassiani et al. (2005a), replace (34) with Equation 35:

$$
\mathrm{t}_{\mathrm{m}}=\mu \frac{\sigma_{\mathrm{r}}}{\sigma_{\mathrm{ur}}}
$$

The plume spread $\left(\sigma_{\mathrm{r}}\right)$ is defined as the standard deviation of the particle positions, with respect to the barycentre of the corresponding instantaneous plumes, in case of homogeneous and isotropic turbulence. This definition has been extended to non-homogeneous and non-isotropic turbulence, assuming this parameter to represent the spatial scale which quantifies the dimensions of the instantaneous plumes. The standard deviation of the relative velocity $\left(\sigma_{\mathrm{ur}}\right)$ is the mean root square of the difference between the particle velocity and the velocity at the barycentre of its instantaneous corresponding plume. Even this definition is extended to non-homogeneous and non-isotropic turbulence. $\mu$ is an empirical constant, equal to $\mu_{1}=\sqrt{2 / 3}$ for linear continuous source emissions and $\mu_{2}=(0.8) \mu_{1}$ for point continuous sources. The plume spread is then computed as follows Equation 36:

$$
\begin{gathered}
\sigma_{\mathrm{r}}^{2}=\frac{\sigma_{\Delta}^{2}}{1+\frac{\left(\sigma_{\Delta}^{2}-\sigma_{0}^{2}\right)}{\left(\sigma_{0}^{2}+2 \sigma^{2} \mathrm{~T}_{\mathrm{L}} \mathrm{t}_{\mathrm{f}}\right)}}, \\
\sigma_{\mathrm{r}}^{2}(\mathrm{t}=0)=\sigma_{0}^{2}, \quad \sigma_{0}^{2}=\frac{2}{3} \mathrm{D}^{2}
\end{gathered}
$$

where, $\mathrm{D}$ is the source length scale. The separation $\sigma_{\Delta}$ is expressed as follows Equation 37: 


$$
\begin{aligned}
& \sigma_{\Delta}^{2}\left(\mathrm{t}_{\mathrm{f}}+\Delta \mathrm{t}\right)=\sigma_{\Delta}^{2}\left(\mathrm{t}_{\mathrm{f}}\right)+3 \mathrm{C}_{\mathrm{r}} \varepsilon\left(\mathrm{t}_{0}+\mathrm{t}_{\mathrm{f}}\right)^{2} \Delta \mathrm{t}, \\
& \sigma_{\Delta}^{2}\left(\mathrm{t}_{\mathrm{f}}=0\right)=\sigma_{0}^{2}, \quad \mathrm{t}_{0} \equiv\left(\frac{\sigma_{0}^{2}}{\mathrm{C}_{\mathrm{r}} \varepsilon}\right)^{\frac{1}{3}}
\end{aligned}
$$

The instantaneous plume spread grows with the fly time, depends on the source dimension, the Lagrangian time scale and the dissipation rate $\varepsilon$ of the turbulent kinetic energy q. $\mathrm{C}_{\mathrm{r}}$ is the Richardson constant (assumed to be 0.3 in (Cassiani et al., 2005a).

$\sigma_{\mathrm{ur}}$ is related to the plume spread, $\mathrm{q}$ and $\varepsilon$, according to Equation 38:

$$
\begin{aligned}
& \sigma_{u r}^{2}=\frac{2}{3} q\left(\frac{\sigma_{r}}{L}\right)^{\frac{2}{3}}, \\
& L \equiv \frac{(q)^{\frac{3}{2}}}{\varepsilon}, \quad q=\frac{\left(\sigma_{u}^{2}+\sigma_{v}^{2}+\sigma_{w}^{2}\right)}{2}
\end{aligned}
$$

Introducing (36), (37) and (38) in (35), the mixing time scale can be even expressed in the form Equation 39:

$$
\mathrm{t}_{\mathrm{m}}=\sqrt{\frac{3}{2}} \mu \frac{\left(\sigma_{\mathrm{r}}\right)^{\frac{2}{3}}}{\varepsilon^{\frac{1}{3}}}
$$

Equation 36 is consistent with the similarity theory of the relative dispersion (Cassiani et al., 2005a) in that $\sigma_{\mathrm{r}}=$ $\sigma_{0}$ for $\mathrm{t}_{\mathrm{f}} \rightarrow 0, \sigma_{\mathrm{r}}=\sigma_{\Delta}$ for $\mathrm{t}_{\mathrm{s}} \ll<\mathrm{t}_{\mathrm{f}}<<\mathrm{T}_{\mathrm{L}}$, where $t_{s} \equiv\left(\sigma_{0}^{2} / \varepsilon\right)^{1 / 3}$ and $\sigma_{r}=\sqrt{2 \sigma^{2} T_{L} t_{f}}$ for $t_{f}>T_{L}$, where $\sigma^{2}=$ $(2 / 3) q$. The corresponding values for $t_{m}$ are Equation 40:

$$
\begin{aligned}
& \mathrm{t}_{\mathrm{m}}=\mu_{\mathrm{i}} \sqrt{\frac{3}{2}}\left(\frac{\sigma_{0}^{2}}{\varepsilon}\right)^{\frac{1}{3}}, \quad \mathrm{t}_{\mathrm{f}} \rightarrow 0 \\
& \mathrm{t}_{\mathrm{m}}=\mu_{\mathrm{i}} \sqrt{\frac{3}{2}}\left(\mathrm{C}_{\mathrm{r}}\right)^{\frac{1}{3}} \mathrm{t}_{\mathrm{f}} \cong \mathrm{bt}_{\mathrm{f}}, \quad \mathrm{t}_{\mathrm{s}}<<\mathrm{t}_{\mathrm{f}}<<\mathrm{T}_{\mathrm{L}} \\
& \mathrm{t}_{\mathrm{m}}=\mu_{\mathrm{i}} \sqrt{2 \mathrm{~T}_{\mathrm{L}} \mathrm{t}_{\mathrm{f}}}, \quad \mathrm{t}_{\mathrm{f}} \gg \mathrm{T}_{\mathrm{L}}
\end{aligned}
$$

The second equation follows from the comparison with equation (34) and the last equation is derived with the constraint $\sigma_{\text {ur }}=\sigma$ (Cassiani et al., 2005a). Close to the source $t_{m}$ does not depend on the fly time. For intermediate times (but much smaller than $T_{L}$ ) $t_{m}$ is linear in $t_{f}$. When the fly time is much greater than $T_{L}, t_{m}$ goes with $\sqrt{t_{f}}$ (far field). Finally the computation of $\sigma_{r}$ is performed during the macromixing phase, considering the characteristics of a sub-ensemble of the macromixing particles.

\subsection{The Mixing Time Scale: Simpler Formulations}

A simpler and faster alternative to (35) was presented in (Amicarelli et al., 2011b) (after a first mention in (Amicarelli et al., 2008) Equation 41:

$\mathrm{t}_{\mathrm{m}}=\mu\left[\left(\frac{3}{2}\right)^{1 / 2} \frac{\sigma_{0}^{2 / 3}}{\varepsilon^{1 / 3}}+\sqrt{2 \mathrm{~T}_{\mathrm{L}} \mathrm{t}_{\mathrm{f}}}\right]$

The asymptotic behaviour of this formulation is compatible with the first and the last limits of (40). This equation makes the macromixing process faster, as the only additional parameter required is the fly time. On the other hand, however, the relationship between $t_{m}$ and $t_{f}$ is not linear in the mid-field.

Another formulation would constrain the formulation to the first two limits of (40), as follows (providing $\mathrm{C}_{\mathrm{r}}$ ) Equation 42:

$$
\mathrm{t}_{\mathrm{m}}=\mu\left[\left(\frac{3}{2}\right)^{1 / 2} \frac{\sigma_{0}^{2 / 3}}{\varepsilon^{1 / 3}}+\sqrt{\frac{3}{2}}\left(\mathrm{C}_{\mathrm{r}}\right)^{\frac{1}{3}} \mathrm{t}_{\mathrm{f}}\right]
$$

\subsection{The Mixing Time Scale in Homogeneous Turbulence Mixing and Uniform Scalar Gradient}

IECM and IEM provide the same results under homogenous turbulence mixing, with an uniform scalar gradient. Under these conditions the formulation for the mixing time scale, reported by Pope (2000), can be used Equation 43:

$\mathrm{t}_{\mathrm{m}}=\frac{2 \mathrm{q}}{\mathrm{C}_{\varphi} \varepsilon}$

The constant $\mathrm{C}_{\Phi}$ is usually equal to 2 . In these regions (far field with slight scalar gradients) all the turbulent vortices are involved in the mixing phenomenon. Further, this formulation can be used as an upper limit for $\mathrm{t}_{\mathrm{m}}$, as provided by $(34,35$ or 42$)$.

For multi-source phenomena we should estimate a mixing time for each source (Luhar and Sawford, 2005a). A unique value of $t_{m}$ can then be roughly used, averaging all the source dependent estimations. 
The influence of the molecular diffusion coefficient is implicitly taken into account in $t_{\mathrm{m}}$ formulation, as $\mu$ and $b$ depends on the main flow type. These parameters are even sensitive to the boundary layer type and the geometry of the source. The dependency on temperature and the pollutant species are instead usually neglected.

\subsection{IECM Scheme for Reactive Pollutants}

The IECM scheme can be adapted in order to represent pollutant transformations. In the case of a reactant (species $A$ ), subjected to a second order kinetic reaction $\left(-\mathrm{rC}_{\mathrm{A}} \mathrm{C}_{\mathrm{B}}\right)$, IECM takes the following form ( $\mathrm{r}$ : reaction rate for $A)$ Equation 44:

$$
\begin{aligned}
& \frac{\mathrm{dC}_{\mathrm{A}}}{\mathrm{dt}}=-\frac{\mathrm{C}_{\mathrm{A}}-\left\langle\mathrm{C}_{\mathrm{A}} \mid \underline{\mathrm{U}}\right\rangle}{\mathrm{t}_{\mathrm{m}}}-\mathrm{rC}_{\mathrm{A}} \mathrm{C}_{\mathrm{B}} \\
& \frac{\mathrm{dC} \mathrm{C}_{\mathrm{A}}}{\mathrm{dt}}=\mathrm{C}_{\mathrm{A}}\left(-\frac{1}{\mathrm{t}_{\mathrm{m}}}-\mathrm{rC}_{\mathrm{B}}\right)+\frac{\left\langle\mathrm{C}_{\mathrm{A}} \mid \underline{\mathrm{U}}\right\rangle}{\mathrm{t}_{\mathrm{m}}} \Rightarrow \\
& \Rightarrow \int_{\mathrm{C}_{\mathrm{A}}(\mathrm{t})}^{\mathrm{C}_{\mathrm{A}}(\mathrm{t}+\mathrm{dt})} \frac{\mathrm{dC}_{\mathrm{A}}}{\left(\mathrm{aC} \mathrm{C}_{\mathrm{A}}+\mathrm{b}\right)}=\int_{\mathrm{t}}^{\mathrm{t}+\mathrm{dt}} \mathrm{dt}, \\
& \mathrm{a} \equiv\left(-\frac{1}{\mathrm{t}_{\mathrm{m}}}-\mathrm{rC}_{\mathrm{B}}\right), \quad \mathrm{b} \equiv \frac{\left\langle\mathrm{C}_{\mathrm{A}} \mid \underline{\mathrm{U}}\right\rangle}{\mathrm{t}_{\mathrm{m}}} \\
& \left.\frac{1}{\mathrm{a}} \ln \left(\mathrm{aC} \mathrm{C}_{\mathrm{A}}+\mathrm{b}\right)\right]_{\mathrm{C}_{\mathrm{A}}(\mathrm{t})}^{\mathrm{C}_{\mathrm{A}}(\mathrm{t}+\mathrm{dt})}=\mathrm{dt} \Rightarrow \\
& \mathrm{aC} \mathrm{C}_{\mathrm{A}}(\mathrm{t}+\mathrm{dt})+\mathrm{b}=\mathrm{e}^{\mathrm{adt}}\left(\mathrm{aC} \mathrm{C}_{\mathrm{A}}(\mathrm{t})+\mathrm{b}\right) \Rightarrow \\
& \Rightarrow \mathrm{C}_{\mathrm{A}}(\mathrm{t}+\mathrm{dt})=\mathrm{C}_{\mathrm{A}}(\mathrm{t}) \mathrm{e}^{-\mathrm{dt}\left(\frac{1}{\mathrm{t}_{\mathrm{m}}}+\mathrm{rC}_{\mathrm{B}}\right)}+ \\
& +\frac{\left\langle\mathrm{C}_{\mathrm{A}} \mid \underline{\mathrm{U}}\right\rangle}{\left(\mathrm{rt}_{\mathrm{m}} \mathrm{C}_{\mathrm{B}}+1\right)}\left(1-\mathrm{e}^{-\mathrm{dt}\left(\frac{1}{\mathrm{t}_{\mathrm{m}}}+\mathrm{rC}_{\mathrm{B}}\right)}\right)
\end{aligned}
$$

This relation requires the time step to be much smaller than $t_{m}$ and the reaction time scale, in order to consider $\langle\mathrm{C} \mid \underline{\mathrm{U}}\rangle, \mathrm{C}_{\mathrm{B}}$ (the particle concentration of the coreactant) and $t_{m}$ constant during the time step. An advantage of IECM is that the reactive terms do not need any additional computation. So that we can model multispecies and multi-reactions involved in complex pollutant dispersion phenomena, no matter about the reaction order, just using an analytical equation. In particular, concentration fluctuations can influence the estimation of the mean balance equation (above example) Equation 45:

$$
\overline{\mathrm{dC}}=\overline{\mathrm{dt}}, \quad \overline{\mathrm{T}}=-\mathrm{r}\left(\overline{\mathrm{C}_{\mathrm{A}}} \cdot \overline{\mathrm{C}_{\mathrm{B}}}+\overline{\mathrm{C}_{\mathrm{A}}^{\prime} \mathrm{C}_{\mathrm{B}}^{\prime}}\right)
$$

In order to quantify the importance of concentration fluctuations under these conditions, the segregation coefficient is normally evaluated Equation 46:

$$
\mathrm{I}_{\mathrm{s}} \equiv \frac{\overline{\mathrm{C}_{\mathrm{A}}^{\prime} \mathrm{C}_{\mathrm{B}}^{\prime}}}{\overline{\mathrm{C}_{\mathrm{A}}} \cdot \overline{\mathrm{C}_{\mathrm{B}}}}
$$

On the other hand an alternative approach has been adopted with two reactive pollutants. Sawford (2006a) derives the reactive terms as functions of the "mixture fraction". This parameter depends on the difference between the concentrations of the two reactants, which is a passive scalar. Then this can be computed as an additional passive and fictitious species, during the macromixing phase.

\subsection{Simplified algorithms}

Several simplifications of the generic algorithm discussed at the beginning of Sec. 2 have been validated, in order to further reduce the computational costs, according to the particular class of phenomena to be represented.

When the mean plume is compact and a prevailing mean flow direction is present, the following procedure can be adopted for passive pollutants (Amicarelli et al., 2011b; Cassiani et al., 2007a; Leuzzi et al., 2012), sequentially coupling a macromixing and a micromixing schemes, as described in the following. According to this procedure, we do not need to model the turbulent flow where the instantaneous concentration is null.

During the macromixing phase particles can be released only from the sources, as clean (conservative) particles do not contribute to the mean concentration field. During this phase $\Delta t$ should be at least one order of magnitude lower than $T_{L}$ and the ratio between the grid resolution $\Delta \mathrm{x}$ and the velocity scale of the main flow. During this phase the concentration means are computed, so are the other parameters required by the micromixing scheme.

During the micromixing phase the simulation runs again from the beginning. Particles are released only from the up-flow edge of the plume and move according to the macromixing scheme. The mean concentrations are not computed anymore, but used in the micromixing equations. The higher moments of concentration are then estimated.

\section{LAGRANGIAN MICROMIXING MODELS: VALIDATIONS}

The first published studies on complete Lagrangian micromixing models seem to date back to 2004 
(Sawford, 2004a). As far several research groups have been working on the subject. This section tries to synthesize some numerical solutions, which have been successfully investigated and validated. A corresponding overview is reported in Table 2.

Lagrangian micromixing models have been validated on pollutant dispersion phenomena in homogeneous turbulence, grid turbulence, neutral boundary layers, even with canopy (vegetation or streetcanyons) and convective boundary layers. These studies refer to 2D models, even if some codes have been developed in 1D and 3D (Amicarelli et al., 2011a; Postma et al., 2011b; Leuzzi et al., 2012).

Kolmogorov constant usually varies between 1.5 and 5, according to the particular boundary layer configuration. IECM seems to represent the most efficient micromixing scheme, as resulting from intercomparisons (Sawford, 2004a; Cassiani et al., 2007b; Amicarelli et al., 2011b; Leuzzi et al., 2012). The micromixing constants for the mid-field usually varies within the ranges $0.3 \leq \mu \leq 0.8$ and $0.6 \leq b \leq 1.2$, depending on the source configuration and the boundary layer type. The micromixing constant for homogeneous turbulence varies between 1.8 and 3.0 , usually taken as 2 . The highest simulated values of the intensity of fluctuations reach $10^{3}$. The absolute value of skewness can arise up to 80 and kurtosis up to $10^{4}$. The peak concentration can be up to $10^{2}-10^{3}$ times greater than the mean. Further the performances of these models have been sometimes investigated in terms of validation metrics (Leuzzi et al., 2012), according to (VDI, 2005; Chang and Hanna, 2004).

Generally Lagrangian micromixing models show several advantages, with respect to other numerical models for concentration fluctuations. They usually provide:

- Limited computational costs

- Neither convergence algorithm, nor computational mesh

- An unique (micromixing) scheme for every statistical moment of concentration and a direct estimation of $\mathrm{f}_{\mathrm{C}}$

- Reliable modelling of the effect of the velocity autocorrelation (estimation of trajectories)

- A reliable formulation of the mixing time scale in the near- and mid- field, depending on the fly time

- A more accurate modelling of the pollutant reactions, related to the instantaneous (particle) concentrations

On the other hand the Lagrangian micromixing shortcomings can be synthesised as follows:
- Transformations in 3D, complex emissions processes topography or bathymetry, buoyant or non-buotant pollutants, non-stationary velocity statistics have not yet been investigated, because of the recent development of the models

- Lagrangian micromixing models need the Eulerian velocity statistics of the main flow in input from another code or a diagnostic tool (nevertheless they can be directly coupled to RANS models)

The validation studies of the Lagrangian micromixing models and the corresponding experimental databases show the importance of concentration fluctuations for micro-scale pollutant dispersion phenomena, even for passive pollutants and in presence of obstacles or street canyons.

Finally Lagrangian micromixing models have been recently coupled with Eulerian stochastic models in order to reproduce concentration time series. In this context (Cassiani et al., 2009) they have been used to provide the conditional mean to an Eulerian stochastic model for concentration time series.

\section{CONCLUSION}

Lagrangian micromixing models have been recently developed and applied to simulate pollutant dispersion phenomena, in order to estimate both the mean and the turbulent fluctuations of concentration. This study represents a synthetic overview of the main characteristics of these models, their numerical schemes and algorithms, furnishing a simple reference for model developers. The most rigorous macromixing scheme, used to estimate particle trajectories, was developed by Thomson (1987) and several simplified schemes have been proposed in literature. With regard to micromixing schemes, the theoretical advantages of IECM respect to IEM have been discussed. Some formulations of the mixing time, consistent with the similarity theory of the relative dispersion, have been presented. Furthermore, an IECM scheme for two reactive species has been proposed. The analysis of the case studies performed for the validation of the Lagrangian micromixing schemes suggests that this approach represents an efficient numerical tool, in order to model the mean concentrations and their fluctuations. The corresponding application fields refer to several dispersion phenomena such as: accidents (power or production plants, terroristic attacks, hydrocarbons storage and transport, extraordinary emissions), odours (power plants and 
energy production from waste-compost, dumps, incinerators, biogas storage and smokes-, high enthalpy geothermic plants -sulfide hydrogen-, animal farms), micro-scale dispersion from continuous or spot emissions (traffic pollutants, power or production plants, dispersion in aquatic environments...), industrial processes (combustion, pollutant treatment,...), strong non-linear relationship between concentration and damage (inflammable substances, explosions,..), reactions depending on instantaneous concentrations.

\section{ACKNOWLEDGEMENT}

The researchers are deeply grateful to Prof. Brian Sawford, who provided comments and advices during the early stage of this study.

This study has been financed by the Research Fund for the Italian Electrical System under the Contract Agreement between ERSE and the Ministry of Economic Development-General Directorate for Energy and Mining Resources stipulated on 29 July 2009 in compliance with the Decree of 19 March 2009.

\section{REFERENCES}

Amicarelli, A., 2009. Sviluppo di modelli numerici lagrangiani di micromixing per la stima della media e delle fluttuazioni della concentrazione. P.hD Thesis, Biblioteca Nazionale Centrale di Firenze, Archivio Regionale del Lazio.

Amicarelli, A., G. Leuzzi and P. Monti, 2008. A stationary 3D Lagrangian stochastic numerical model for concentration fluctuations. Croatian Meteorol. J., 43: 387-391.

Amicarelli, A., G. Leuzzi, P. Monti and D.J. Thomson, 2011a. A comparison between IECM and IEM Lagrangian models. Int. J. Environ. Pollut., 44: 324-331.

Amicarelli, A., G. Leuzzi, P. Monti and D.J. Thomson, 2011b. LAGFLUM, a stationary 3D Lagrangian stochastic numerical micromixing model for concentration fluctuations: Validation in canopy turbulence, on the MUST wind tunnel experiment. Int. J. Environ. Pollut., 47: 317-325. DOI: 10.1504/IJEP.2011.047345

Amicarelli, A., P. Salizzoni, G. Leuzzi, P.Monti and L. Soulhac et al., 2012. Sensitivity analysis of a concentration fluctuation model to dissipation rate estimates. Int. J. Environ. Pollut.

Bezpalcova, K., 2007. Physical modelling of flow and diffusion in urban canopy. P.hD Thesis, Charles University, Prague.
Cassiani, M., A. Radicchi and A.J. lbertson, 2007a. Modelling of concentration fluctuations in canopy turbulence. Boundary-Layer Meteorol., 122: 655681. DOI: 10.1007/s10546-006-9122-0

Cassiani, M., A. Radicchi, J.D. Albertson and U. Giostra, 2007b. An efficient algorithm for scalar pdf modelling in incompressible turbulent flow; numerical analysis with evaluation of IEM and IECM micro-mixing models. J. Comput. Phys., 223: 519-550. DOI: 10.1016/j.jcp.2006.09.023

Cassiani, M., P. Franzese and U. Giostra, 2005a. A PDF micromixing model of dispersion for atmospheric flow. Part I: Development of the model, application to homogeneous turbulence and ti neutral boundary layer. Atmos. Environ., 39: 1457-1469. DOI: 10.1016/j.atmosenv.2004.11.020

Cassiani, M., P. Franzese and U. Giostra, 2005b. A PDF micromixing model of dispersion for atmospheric flow. Part II: Application to convective boundary layer. Atmos. Environ., 39: 1471-1479. DOI: 10.1016/j.atmosenv.2004.11.019

Cassiani, M., A. Radicchi and U. Giostra, 2005c. Probability density function modelling of concentration fluctuation in and above a canopy layer. Agric. Forest Meteorol., 133: 153-165. DOI: 10.1016/j.agrformet.2005.09.007

Cassiani, M., P. Franzese and J.D. Albertson, 2009. A coupled Eulerian and Lagrangian mixing model for intermittent concentration time series. Phys. Fluids, 21: 085105085105. DOI: 10.1063/1.3202534

Chang, J.C. and S.R. Hanna, 2004. Air quality model performance evaluation. Meteorol. Atmos. Phys., 87: 167-196. DOI: 10.1007/s00703-003-0070-7

Chatwin, P.C. and C. Robinson, 1997. The moments of the PDF of concentration for gas clouds in the presence of fences. Il Nuovo Cimento.

Chatwin, P.C., D.M. Lewis and N. Mole, 1997. Comments on the properties and uses of atmospheric dispersion datasets. IL Nuovo Cimento, $2^{\circ} \mathrm{C}$, 3: 475-489.

Csanady, G.T., 1967. Concentration fluctuations in turbulent diffusion. J. Atmos. Sci., 24: 21-28. DOI: $10.1175 / 1520-$ 0469(1967)024<0021:CFITD>2.0.CO;2

Dixon, N.S. and A.S. Tomlin, 2007. A Lagrangian stochastic model for predicting concentration fluctuations in urban areas. Atmos. Environ., 41: 8114-8127. DOI: 10.1016/j.atmosenv.2007.06.033

Dopazo, C. and E.E. O'Brien, 1974. An approach to the autoignition of a turbulent mixture. Acta Astronautica, 1: 1239-1266. DOI: 10.1016/00945765(74)90050-2 
Fackrell, J.E. and A.G. Robins, 1982. Concentrationf luctuations and fluxes in plumes from point sources in a turbulent boundary layer. J. Fluid Mech., 117: 1-26. DOI: 10.1017/S0022112082001499

Klein, P.M. and D.T. Young, 2011. Concentration fluctuations in a downtown urban area. Part I: Analysis of Joint Urban 2003 full-scale fastresponse measurements. Environ. Fluid Mech., 11: 23-42. DOI: $10.1007 / \mathrm{s} 10652-010-9194-8$

Klein, P.M., B. Leitl and M. Schatzmann, 2011. Concentration fluctuations in a downtown urban area. Part II: Analysis of Joint Urban 2003 windtunnel measurements. Environ. Fluid Mech., 11: 4360. DOI: $10.1007 / \mathrm{s} 10652-010-9195-7$

Leuzzi, G., A. Amicarelli, P. Monti and D.J. Thomson, 2012. A 3D Lagrangian micromixing dispersion model LAGFLUM and its validation with a wind tunnel experiment. Atmos. Environ., 54: 117-126. DOI: 10.1016/j.atmosenv.2012.02.054

Luhar, A.K. and B.L. Sawford, 2005a. Micromixing modelling of mean and fluctuating scalar fields in the convective boundary layer. Atmos. Environ., 39: 6673-6685. DOI: 10.1016/j.atmosenv.2005.07.047

Luhar, A.K. and B.L. Sawford, 2005b. Micromixing modelling of concentration fluctuations in inhomogeneous turbulence in the convective boundary layer. Boundary-Layer Meteorol., 114: 130. DOI: $10.1007 / \mathrm{s} 10546-004-1292-\mathrm{Z}$

Luhar, A.K. and R.E. Britter, 1989. A random walk model for dispersion in inhomogeneous turbulence in a convective boundary layer. Atmos. Environ., 23: 1911-1924. DOI: 10.1016/0004-6981(89)90516-7

Mah, B. K. and Z. Warhaft, 1986. Some aspects of the thermal mixing layer in grid turbulence. Phys. Fluids, 29: 3114-3120. DOI: 10.1063/1.865962

Mylne, K., M.J. Davidson and D.J. Thomson, 1995. Concentration fluctuation measurements in tracer plumes using high and low frequency response detectors. Boundary-Layer Meteorol., 79: 225-242. DOI: 10.1007/BF00119439

Pavageau, M. and M. Schatzmann, 1999. Wind tunnel measurements of concentration fluctuations in an urban street canyon. Atmos. Environ., 33: 39613971. DOI: 10.1016/S1352-2310(99)00138-7

Pope, S.B., 1998. The vanishing effect of molecular diffusivity on turbulent dispersion: Implications for turbulent mixing and the scalar flux. J. Fluid Mech., 359: 299-312.

Pope, S.B., 2000; Turbulent Flows. 1st Edn., Cornell University, New York, ISBN-10: 0521598869, pp: 771.
Postma, J.V., J.D. Wilson and E. Yee, 2011a. Comparing two implementations of a micromixing model. Part I: Wall shear-layer flow. Boundary-Layer Meteorol., 140: 207-224. DOI: 10.1007/s10546-011-9605-5

Postma, J.V., J.D. Wilson and E. Yee, 2011b. Comparing Two Implementations of a Micromixing Model. Part II: Canopy Flow. Boundary-Layer Meteorol., 140: 225-241. DOI: 10.1007/s10546-011-9606-4

Sawford, B.L., 2004a. Micro-Mixing modelling of scalar fluctuations for plumes in homogeneous turbulence. Flow, Turbulence Comb., 72: 133-160. DOI: 10.1023/B:APPL.0000044409.74300.db

Sawford, B.L., 2004b. Conditional scalar mixing statistics in homogeneous isotropic turbulence. J. Phys., 6: 55-55. DOI: 10.1088/1367-2630/6/1/055

Sawford, B.L., 2006a. Lagrangian stochastic modelling of chemical reaction in a scalar mixing layer. Boundary-Layer Meteorol., 118: 1-23. DOI: 10.1007/s10546-005-4737-0

Sawford, B.L., 2006b. Lagrangian modeling of scalar statistics in a double scalar mixing layer. Phys. Fluids, 18: 085108-085108. DOI: 10.1063/1.2335154

Sykes, R.I., W.S. Lewellen and S.F. Parker, 1984. A turbulent-transport model for concentration fluctuations and fluxes. J. Fluid Mech., 139: 193218. DOI: $10.1017 / \mathrm{S} 002211208400032 \mathrm{X}$

Thomson, D.J., 1987. Criteria for the selection of stochastic models of particle trajectories in turbulent flows. J. Fluid Mech., 180: 529-556. DOI: 10.1017/S0022112087001940

VDI, 2005. Environmental meteorology-Prognostic microscale windfield models-Evaluation for flow around buildings and obstacles. Verein Deutscher Ingenieure guideline, 3783, Part 9, Beuth, Berlin.

Villermaux, J. and J.C. Devillon, 1972. Representation de la coalescence et de la redispersion des domains de segregation dans un fluide par un modèle d'interaction phenomenologique. Proceedings of ths 2nd International Symposium on Chemical Reaction Engineering, (CRE' 72), Elsevier, New York.

Yee, E. and C.A. Biltoft, 2004. Concentration fluctuation measurements in a plume dispersing through a regular array of obstacles. Boundary-Layer Meteorol., 111: 363-415. DOI: 10.1023/B:BOUN.0000016496.83909.ee

Yee, E., R. Chan, P.R., Kosteniuk, G.M. Chandler and C.A. Biltoft et al., 1994. Experimental measurements of concentration fluctuations and scales in a dispersing plume in the atmospheric surface layer obtained using a very fast response concentration detector. J. Applied Meteorol., 33, 996-1016. DOI: 10.1175/15200450(1994)033<0996:EMOCFA $>2.0 . \mathrm{CO} ; 2$ 